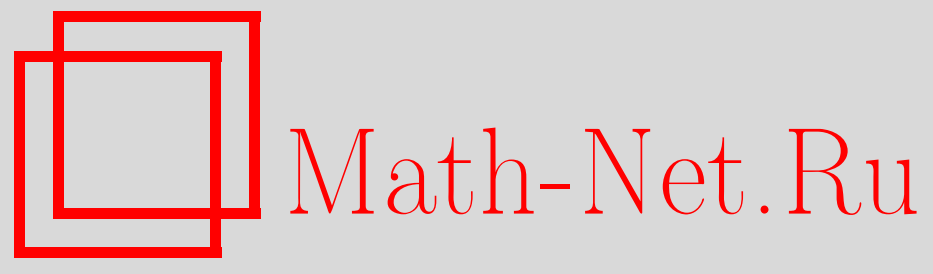

М. Н. Назаров, Математическое моделирование тканеобразования на основе систем дифференциальных уравнений, Вестн. Сам. гос. техн. ун-та. Сер. Физ.-мат. науки, 2017, номер 3, 581-594

DOI: https://doi.org/10.14498/vsgtu1535

Использование Общероссийского математического портала MathNet.Ru подразумевает, что вы прочитали и согласны с пользовательским соглашением

http://www. mathnet.ru/rus/agreement

Параметры загрузки:

IP: 3.91 .87 .62

26 апреля 2023 г., 08:02:00

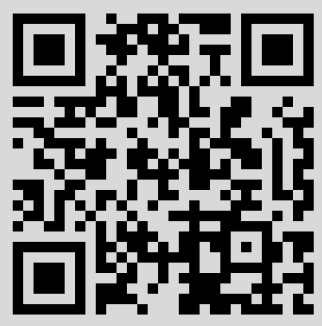


Вестн. Сам. гос. техн. ун-та. Сер. Физ.-мат. науки. 2017. Т. 21, № 3. С. $581-594$ ISSN: 2310-7081 (online), 1991-8615 (print)

УДК 517.958:57

\title{
Математическое моделирование тканеобразования на основе систем дифференциальных уравнений
}

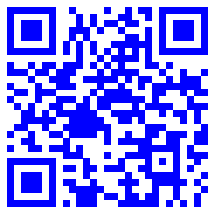

\author{
М. Н. Назаров \\ Национальный исследовательский университет «МИЭТ», \\ Россия, 124498, Москва, Зеленоград, пл. Шокина, 1.
}

\section{Аннотация}

Предложена математическая модель для описания популяционной динамики клеточных скоплений на основе систем обыкновенных дифференциальных уравнений первого порядка. Главным требованием при построении уравнений модели являлось наличие формального биологического обоснования для их вывода, а также доказательство их корректности. Дополнительно к этому для всех параметров, задействованных в уравнениях, было потребовано наличие биологического смысла, а также возможность их оценки либо в ходе эксперимента, либо с помощью моделей внутриклеточной биохимии. При построении искомой модели в качестве основного механизма для координации роста ткани и выбора клетками новых типов при делении был выбран межклеточный обмен специальными сигнальными молекулами. Для упрощения все сигнальные молекулы, которые способны создавать клетки одного типа, не рассматривались по отдельности в модели, а объединялись в виде единого комплекса молекул - «обобщенного сигнала». Подобный подход позволяет в итоге задавать сигналы как функции от типов клеток и вводить в модели их воздействия в виде матриц, где строки отвечают за типы клеток, принимающих сигналы, а столбцы - за типы клеток, испускающих сигналы.

Ключевые слова: моделирование морфогенеза, обыкновенные дифференциальные уравнения, системная биология, иерархические модели.

Получение: 22 марта 2017 г. / Исправление: 13 июня 2017 г.

Принятие: 18 сентября 2017 г. / Публикация онлайн: 20 сентября 2017 г.

\section{Статья}

( () (7) Контент публикуется на условиях лицензии Creative Commons Attribution 4.0 International (https://creativecommons.org/licenses/by/4.0/deed.ru)

\section{Образец для цитирования}

Н аз а р в В. Н. Математическое моделирование тканеобразования на основе систем дифференциальных уравнений // Вестн. Сам. гос. техн. ун-та. Сер. Физ.-мат. науки, 2017. T. 21, № 3. С. 581-594. doi: 10.14498/vsgtu1535.

\section{Сведения об авторе}

Максим Николаевич Назаров (D) http://orcid.org/0000-0001-8064-1374

старший преподаватель; каф. высшей математики-1; e-mail: nazarov-maximilian@yandex.ru 
Введение. Целью данной работы является построение базовой модели для описания популяционной динамики многоклеточных скоплений. Основной задачей разрабатываемой модели является реализация интерфейса между моделями внутриклеточной биохимии и моделями морфогенеза. Для решения этой задачи налагаются определенные ограничения на уравнения и параметры, которые используются в модели. В первую очередь постулируется, что все уравнения должны иметь строгое биологическое обоснование, а параметры - обладать биологическим смыслом и быть доступными для измерения или формализации на основе биохимических внутриклеточных моделей.

Перед тем как переходить к описанию разрабатываемой популяционной модели, приведем обзор различных подходов, которые были выработаны для моделирования морфогенеза, и поясним, почему ни один из них нам не подходит. Весь существующий набор моделей морфогенеза можно условно разбить на два крупных класса: имитационные и количественные.

K первому классу относятся такие модели, которые используют дискретный подход и вводят сами клетки или их субъединицы в явном виде. Примерами имитационных моделей являются: модель клеточных автоматов [1], сфероидная модель [2-4] и клеточная модель Поттса [5-7]. Модели данного класса мы не сможем использовать в качестве базовых моделей тканеобразования главным образом по причине высокой вычислительной сложности их реализации.

В свою очередь, количественные модели дают усредненное описание динамики клеток на основе систем дифференциальных уравнений. В частности, скопление клеток может быть описано в рамках данного подхода как эластичный материал с помощью тензора деформации $[8,9]$ либо как вязкая жидкость [10,11], и, в том числе, с помощью уравнений Навье-Стокса [12]. Вариант с тензором деформации мог бы нас устроить, если бы удалось построить внутриклеточную модель, которая связала бы внутриклеточную биохимию, генные сети и описание деформаций цитоскелета. Для второго варианта также требуется внутриклеточная модель, которая связала бы генные сети, биохимию и клеточную адгезию. При отсутствии этих инструментов мы не сможем построить базовую модель на основе этого подхода. Другой вариант заключается в моделировании динамики распределения особых сигнальных молекул морфогенов, для описания которой строятся системы уравнений типа «реакция-диффузия» [13]. В чистом виде данный подход требует "ad hoc"-допущений, что делает его недостаточно строгим для наших целей.

Однако можно отказаться от моделирования трехмерного распределения клеток и попробовать построить более простую модель для клеточных популяций, обменивающихся сигнальными молекулами. При этом итоговая модель, очевидно, будет ближе ${ }^{1}$ к классическим популяционным моделям [15], нежели к существующим моделями морфогенеза. В этом случае можно получить более строгую модель, которая удовлетворит нашим требования биологически осмысленных параметров и уравнений со строгим выводом, но при этом не будет слишком сложной для вычислительной реализации.

Для достижения этой цели предлагается использовать набор универсальных параметров, для которых должны выполняться три условия.

\footnotetext{
${ }^{1}$ Система дифференциальных уравнения для численностей клеток.
} 
1. Все универсальные параметры должны иметь биологический смысл.

2. Значения универсальных параметров должны оцениваться с помощью внутриклеточных биохимических моделей или непосредственно на основе эксперимента.

3. Универсальные параметры должны допускать использование как в имитационных, так и в количественных моделях. При этом их значения должны быть свободно переносимы между этими моделями для отдельных организмов.

ЗАмечАниЕ 1. Применение универсальных параметров сугубо специфично в настоящей работе и нехарактерно ни для одного из актуальных на данный момент направлений в моделировании морфогенеза.

ЗАмечание 2. Использование универсальных параметров вместе с формальным обоснованием вывода уравнений позволит позиционировать итоговую модель как базовую для построения более сложных моделей морфогенеза (в том числе и в дискретных имитационных).

В качестве основного механизма для координации роста ткани и выбора клетками новых типов при делении выбран межклеточный сигналъный обмен молекулами морфогенами. Для его описания в терминах универсальных параметров за основу возьмем набор параметров из работы [16]:

- $T=\left\{\tau_{1}, \ldots, \tau_{n}\right\}$ - типы клеток;

- $I_{S}\left(\tau_{1}\right), \ldots, I_{S}\left(\tau_{n}\right)$ - интенсивности синтеза сигналов морфогенов;

- $\operatorname{Pr}\left(\tau_{1}, \tau_{1}\right), \ldots, \operatorname{Pr}\left(\tau_{n}, \tau_{n}\right)$ - приоритеты сигналов; отдельное значение в этой таблице $\operatorname{Pr}\left(\tau_{i}, \tau_{j}\right)$ задает относительный приоритет воздействия от клеток типа $\tau_{j}$ на клетки типа $\tau_{i}(\operatorname{Pr} \geqslant 0)$;

- $D\left(\tau_{1}, \tau_{1}\right), \ldots, D\left(\tau_{n}, \tau_{n}\right)$ - новые типы для дочерних клеток; отдельное значение в этой таблице $D\left(\tau_{i}, \tau_{j}\right)=\tau$ задает новый тип $\tau$, который под действием клеток $\tau_{j}$ будут получать дочерние клетки после деления $\tau_{i}$;

- $O\left(\tau_{1}, \tau_{1}\right), \ldots, O\left(\tau_{n}, \tau_{n}\right)$ - сохранение клеткой своего типа после деления; для данных параметров допускаются только два значения $-\{0,1\} ;$ в случае $O\left(\tau_{i}, \tau_{j}\right)=1$ клетка типа $\tau_{i}$ под действием клеток типа $\tau_{j}$ должна разделиться на одну клетку старого типа $\tau_{i}$ и одну клетку нового типа $\tau=D\left(\tau_{i}, \tau_{j}\right)$ (деление асимметричное); в противном случае, если значение $O\left(\tau_{i}, \tau_{j}\right)=0$, то после деления $\tau_{i}$ обе клетки должны получить новый тип $\tau$ (деление симметричное);

- $v_{d}^{\max }\left(\tau_{1}\right), \ldots, v_{d}^{\max }\left(\tau_{n}\right)$ - максимальные интенсивности деления (или апоптоза); за элементарный такт времени $\mathrm{d} t$ ровно $v_{d}(\tau) N(\tau) \mathrm{d} t$ клеток либо приступят к делению, либо запустят процесс программируемой смерти, где $v_{d}(\tau) \leqslant v_{d}^{\max }(\tau)$;

- $K\left(\tau_{1}, \tau_{1}\right), \ldots, K\left(\tau_{n}, \tau_{n}\right)$ - элементарные приращения скорости $v_{d}$ наступления деления или программируемой смерти под действием сигналов.

ЗАмЕчАниЕ 3. За моделирование межклеточных взаимодействий будут отвечать в первую очередь матричные параметры $\operatorname{Pr}, D, O$ и $K$. В этих матрицах первый индекс задает тип клетки мишени, а второй - тип клетки источника сигнального раздражения.

Обсудим, каким образом возможно в общем случае провести оценку рассмотренных выше параметров на основе моделей внутриклеточной биохимии (например, [17]).

Тип клетки $\tau_{i}$ в рамках биохимической модели можно задать двумя набо- 
рами значений: состояниями ${ }^{2}$ генов и концентрациями регуляторных веществ, а также границами, в пределах которых данные значения меняются.

Пусть клетка типа $\tau$ способна создавать $k$ видов различных сигналов $\left\{S_{1}(\tau), \ldots, S_{k}(\tau)\right\}$. Для определенности положим, что в единицу времени клетка выделяет в среднем: $I_{j}(\tau)$ сигналов $S_{j}(\tau)$, где $j=\overline{1, k}$. Тогда в качестве $I_{S}(\tau)$ мы можем выбрать

$$
I_{S}(\tau)=\min _{j} I_{j}(\tau)
$$

Для отдельного химического сигнала $S_{j}\left(\tau_{*}\right)$ приоритет можно по определению считать равным суммарной биологической активности $B\left(\tau, S_{j}\left(\tau_{*}\right)\right)$ вторичных посредников, ${ }^{3}$ которые были задействованы при передаче раздражения от $S_{j}\left(\tau_{*}\right)$ внутрь клетки типа $\tau$.

Для вычисления $D\left(\tau, \tau_{*}\right)$ нужно либо с помощью биохимической модели воспроизвести воздействие сигналов $S\left(\tau_{*}\right)$ на клетку типа $\tau$ и оценить, в какой тип могут перейти дочерние клетки от $\tau$ после этого воздействия, либо непосредственно поставить модельный эксперимент с реальными клетками и сигнальными молекулами. Похожим образом можно поступить и с определением параметров $O\left(\tau, \tau_{*}\right)$, проверив, будет ли материнская клетка типа $\tau$ менять свой тип после деления (делиться симметрично или асимметрично).

Аналогично можно провести поиск $K\left(\tau, \tau_{*}\right)$, но за тем лишь исключением, что оценивается влияние сигналов на скорость прохождения клеткой $\tau$ контрольных точек клеточного цикла.

1. Описание базовой модели для клеточных популяций. Основными переменными для базовой модели морфогенеза будут:

- $N(\tau, t)$ - численности клеток как функции от типов $\tau$;

- $v_{d}(\tau, t)$ - интенсивности деления (или программируемой смерти). Уравнения для динамики численности клеток $N(\tau, t)$ примут следующий вид:

$$
\frac{\mathrm{d} N(\tau, t)}{\mathrm{d} t}=\operatorname{Surplus}(\tau, N)-\operatorname{Loss}(\tau, N) .
$$

В уравнении (1) величина $\operatorname{Loss}(\tau, N)$ задает потерю в единицу времени клеток типа $\tau$ в результате деления ${ }^{4}$ (или программируемой смерти), а величина $\operatorname{Surplus}(\tau, N)$ задает прирост в единицу времени клеток типа $\tau$. Для величины $\operatorname{Loss}(\tau, N)$ будет справедлива следующая формула:

$$
\operatorname{Loss}(\tau, N)=v_{d}(\tau, t) \cdot N(\tau, t) .
$$

Для исключения клеток, чья численность меньше единицы, ${ }^{5}$ введем приведенные численности:

$$
\hat{N}(\tau)=\sigma(N(\tau)-1+c) N(\tau)
$$

\footnotetext{
${ }^{2} \mathrm{~B}$ простейшем случае состояние гена можно определить как количество актов копирования гена в единицу времени.

${ }^{3}$ Под биологической активностью подразумевается число реакций в единицу времени, которые катализируют вторичные сигнальные посредники.

${ }^{4}$ Клетка будет считаться потерянной даже в том случае, если после деления обе ее дочерние клетки сохраняют исходный тип.

${ }^{5}$ По умолчанию такие клетки считаются полностью вымершими в рамках модели.
} 
В качестве функции $\sigma$ можно использовать как функцию Хевисайда, так и приближенную к ней сигмоиду

$$
\sigma^{c}(x)=1 /\left(1+e^{-x / c}\right), \quad \text { где } 0<c \ll 1 .
$$

Положение 1. Ровно $v_{d}(\tau, t) \hat{N}(\tau, t) \mathrm{d} t$ клеток типа $\tau$ за элементарный промежуток времени $\mathrm{d} t$ будут потенииально делитъся с образованием не более чем $2 v_{d}(\tau, t) \hat{N}(\tau, t) \mathrm{d} t$ новых клеток (см. рис. 1).

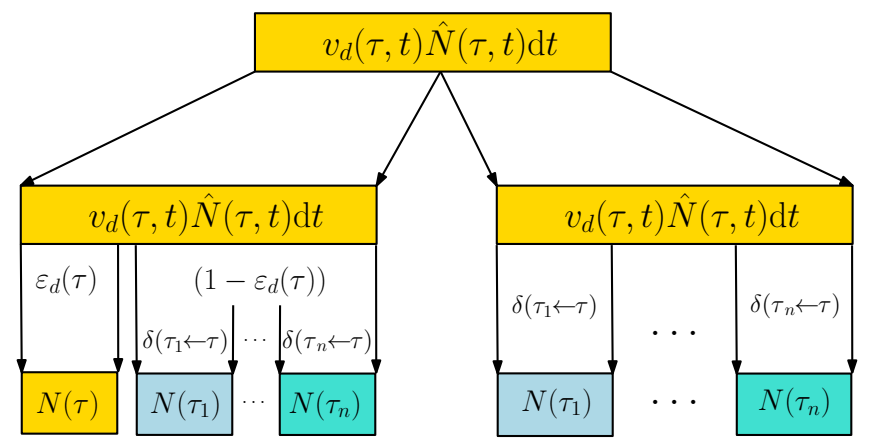

Рис. 1. Деление клеток типа $\tau$ с потенциальным выбором новых типов

[Figure 1 Division of $\tau$-type cells with a potential choice of new types]

ПолоЖениЕ 2. Среди новъх клеток $2 v_{d}(\tau, t) \hat{N}(\tau, t) \mathrm{d} t$ половина будут являться материнскими, а половина - дочерними. Для учета возможности асимметричного деления введем в рассмотрение распределяющии показатель $\varepsilon_{d}(\tau)$, который будет равен относительной доле всех материнских клеток, которье разделилисъ асимметрично. Тогда относительная доля симметрично разделившихся материнских клеток $\tau$ будет равна $\left(1-\varepsilon_{d}(\tau)\right)$.

ПоложениЕ 3. Поскольку часть клеток может в ходе дифферениировки получить новый тип либо погибнуть в результате апоптоза, в рассмотрение вводятся распределяюшие коэфбициенты $\delta\left(\tau_{1} \leftarrow \tau\right) \leqslant 1, \ldots$, $\delta\left(\tau_{M} \leftarrow \tau\right) \leqslant 1$. Каждый отдельный коэфбициент $\delta(\tau \leftarrow \tau)$ задает относительную долю разделившихся клеток типа $\tau$, чъи дочерние клетки получат после деления тип т́.

Если просуммировать число асимметрично разделившихся клеток типа $\tau$, а также число всех тех клеток других типов $\dot{\tau}$, которые дифференцировали в тип $\tau$ после деления, то получится следующая оценка для прироста клеток типа $\tau$ :

$$
\begin{aligned}
& \operatorname{Surplus}(\tau, N)=v_{d}(\tau, t) \hat{N}(\tau, t) \varepsilon_{d}(\tau, N)+ \\
& \quad+\sum_{\dot{\tau}} v_{d}(\dot{\tau}, t) \hat{N}(\dot{\tau}, t) \delta(\tau \leftarrow \dot{\tau}, N)\left(2-\varepsilon_{d}(\dot{\tau}, N)\right) .
\end{aligned}
$$

ПолоЖениЕ 4. За элементарный промежуток времени $\mathrm{d} t$ каждая клетка mипа $\tau$ синтезирует ровно $I_{S}(\tau) \mathrm{d} t$ сигналов. Таких сигналов в отдельныи промежсток в сумме будет $I_{S}(\tau) \hat{N}(\tau, t) \mathrm{d} t$. 
ПолоЖениЕ 5. Постулируем, что в потреблении сигнальных воздействий отсутствует инертность. То есть, все сигналь $I_{S}(\tau) \hat{N}(\tau, t) \mathrm{d} t$, синтезированные клетками типа $\tau$, будут усвоень другими клетками в течении элементарного промежутка $\mathrm{d} t$.

ПоложениЕ 6. Сигналь от любого типа $\tau$ распределяются равномерно между всеми клетками, которые способны принимать эти сигналь.

С учетом данных упрощающих положений суммарное сигнальное воздействие со стороны сигналов типа $\dot{\tau}$ на одну клетку типа $\tau$ будет следующим:

$$
\operatorname{Inf}(\tau \leftarrow \dot{\tau}, N)=\left\{\begin{array}{cll}
\operatorname{Pr}(\tau, \dot{\tau}) \frac{I_{S}(\dot{\tau}) \hat{N}(\dot{\tau})}{\operatorname{Disp}_{\dot{\tau}}(N)}, & \text { если } & \operatorname{Pr}(\tau, \dot{\tau}) \hat{N}(\dot{\tau})>0 \\
0, & \text { если } & \operatorname{Pr}(\tau, \dot{\tau}) \hat{N}(\dot{\tau})=0
\end{array}\right.
$$

В этом выражении коэффициент $\operatorname{Disp}_{\dot{\tau}}(N)$ позволяет учесть распределение сигнального раздражения между всеми клетками, способными принимать данные сигналы (то есть между клетками $\tau^{*}$, для которых $\left.\operatorname{Pr}\left(\tau^{*}, \tau\right)>0\right)$ :

$$
\operatorname{Disp}_{\dot{\tau}}(N)=\max \left\{1, \sum_{\tau^{*}: \operatorname{Pr}\left(\tau^{*}, \dot{\tau}\right)>0} \hat{N}\left(\tau^{*}, t\right)\right\} .
$$

Для описания итогового воздействия на клетки типа $\tau$ нужно в первую очередь учесть конкуренцию между разнотипными сигналами. Это можно сделать, посчитав для начала суммарное сигнальное воздействие на отдельную клетку типа $\tau$ :

$$
\operatorname{SumInf}(\tau, N)=\sum_{\tau} \operatorname{Inf}(\tau \leftarrow \dot{\tau}, N) .
$$

Вклад сигналов типа $\dot{\tau}$ может быть представлен в виде

$$
\operatorname{Inf}(\tau \leftarrow \dot{\tau}, N)=\operatorname{SumInf}(\tau, N) \operatorname{Sig}(\tau \leftarrow \dot{\tau}, N)
$$

(см. рис. 2). При этом отдельный коэффициент $\operatorname{Sig}(\tau \leftarrow \tau, N)$ будет описывать относительный уровень приоритета сигналов от клеток типа $\tau$ в условиях конкуренции с другими сигналами:

$$
\operatorname{Sig}(\tau \leftarrow \dot{\tau}, N)=\left\{\begin{array}{cl}
\frac{\operatorname{Inf}(\tau \leftarrow \dot{\tau}, N)}{\operatorname{SumInf}(\tau, N)}, & \text { если } \operatorname{Inf}(\tau \leftarrow \dot{\tau}, N)>0, \\
0, & \text { если иначе. }
\end{array}\right.
$$

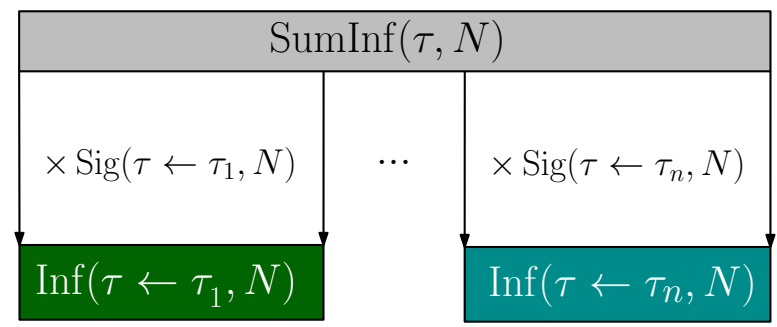

Рис. 2. Распределение суммарного сигнального воздействия на клетки типа $\tau$ [Figure 2. The distribution of the total signaling effect on $\tau$-type cells] 
Величина $K(\tau, \dot{\tau})$ дает приращение в единицу времени скорости $v_{d}(\tau, t)$ под действием сигналов от клеток типа $\dot{\tau}$ в условиях отсутствия конкуренции с другими сигналами и максимальной интенсивности сигнального воздействия. Учитывая конкуренцию между сигналами, а также тот факт, что интенсивность деления (или смерти) $v_{d}(\tau, t)$ не должна становиться меньше нуля или больше $v_{d}^{\max }(\tau)$, получаем уравнение

$$
\begin{aligned}
\frac{\mathrm{d} v_{d}(\tau, t)}{\mathrm{d} t}=\left(1-\frac{v_{d}(t)}{v_{d}^{\max }(\tau)}\right) & \sum_{\tau: K(\tau, \dot{\tau})>0} K(\tau, \dot{\tau}) \operatorname{Sig}(\tau \leftarrow \dot{\tau}, N)+ \\
& +\left(\frac{v_{d}(t)}{v_{d}^{\max }(\tau)}\right) \sum_{\tau: K(\tau, \dot{\tau})<0} K(\tau, \dot{\tau}) \operatorname{Sig}(\tau \leftarrow \dot{\tau}, N) .
\end{aligned}
$$

Относительная доля $\varepsilon_{d}(\tau, N)$ клеток типа $\tau$, которые выберут асимметричное деление, можно посчитать с помощью параметров $O(\tau, \tau)$ и распределяющих коэффициентов $\operatorname{Sig}(\tau \leftarrow \dot{\tau}, N)$ :

$$
\varepsilon_{d}(\tau, N)=\sum_{\tau^{*}: O\left(\dot{\tau}, \tau^{*}\right)=1} \operatorname{Sig}\left(\dot{\tau} \leftarrow \tau^{*}, N\right) .
$$

По аналогии долю $\delta(\tau \leftarrow \tau, N)$ клеток $\tau$, которые дифференцируют в $\tau$ после деления, можно посчитать с помощью параметров $D(\tau, \dot{\tau})$ и $\operatorname{Sig}(\tau \leftarrow \dot{\tau}, N)$ :

$$
\delta(\tau \leftarrow \dot{\tau}, N)=\sum_{\tau^{*}: D\left(\dot{\tau}, \tau^{*}\right)=\tau} \operatorname{Sig}\left(\dot{\tau} \leftarrow \tau^{*}, N\right)
$$

Поскольку коэффициенты $\delta(\tau \leftarrow \tau, N)$ и $\varepsilon_{d}(\tau, N)$ должны задавать распределение долей, для них обязаны выполняться условия нормировки.

$$
\text { УтвеРЖДЕНИЕ. } \forall \dot{\tau} \in T, \quad \sum_{\tau \in T \cup\{0\}} \delta(\tau, \dot{\tau}, N) \leqslant 1 .
$$

Доказательство. Суммируя по всем $\tau$ коэффициенты $\delta(\tau \leftarrow \tau, N)$, получим

$$
\begin{aligned}
& \forall \dot{\tau} \quad \sum_{\tau} \delta(\tau \leftarrow \dot{\tau}, N)=\sum_{\tau} \sum_{\tau^{*}: D\left(\dot{\tau}, \tau^{*}\right)=\tau} \frac{\operatorname{Inf}\left(\dot{\tau} \leftarrow \tau^{*}, N\right)}{\operatorname{SumInf}(\dot{\tau}, N)} \leqslant \\
& \leqslant \frac{1}{\operatorname{SumInf}(\dot{\tau}, N)} \sum_{\tau} \sum_{\tau^{*}: D\left(\dot{\tau}, \tau^{*}\right)=\tau} \operatorname{Inf}\left(\dot{\tau} \leftarrow \tau^{*}, N\right) \leqslant \frac{\operatorname{SumInf}(\dot{\tau}, N)}{\operatorname{SumInf}(\dot{\tau}, N)} \leqslant 1 .
\end{aligned}
$$

АЛГОРИТМ ФОРМАЛИЗАЦИИ И ПРИМЕНЕНИЯ МОДЕЛИ ДЛЯ КОНКРЕТНЫХ ОРГАнизмов.

Шаг 1. Для рассматриваемого организма на основе биохимических моделей или эксперимента оцениваются значения универсальных параметров.

Шаг 2. С использованием данных о развитии ткани корректируются значения параметров, полученные на предыдущем этапе с помощью модели (1)-(10). 
Шаг 3. Построенная в итоге модель для отдельного организма анализируется методами качественного анализа дифференциальных уравнений.

Шаг 4. Значения универсальных параметров, полученные на шаге 2, задействуются в более общей имитационной модели морфогенеза. В качестве начальных конфигураций клеток выбираются те, что будут соответствовать наиболее интересным для нас динамическим режимам, найденным на шаге 3.

ЗАмечАниЕ 3. Важно отметить, что начальные значения для всех универсальных параметров по возможности должны быть получены с помощью биохимических моделей либо экспериментально. Использовать некоторые произвольные приближения для них нерационально, так как модель (1)-(10) не рассчитана на регрессионный подбор параметров. Применение алгоритмов минимизации для них оправдано только для достаточно малых значений вариации этих параметров и при условии хороших начальных приближений. В общем случае данное ограничение выступает существенным лимитирующим фактором, поскольку получение этих данных в ходе экспериментов будет достаточно трудоемкой задачей на текущем уровне развития экспериментальной науки.

ЗАмЕчАНИЕ 4. Важной особенностью итоговой модели является использование коэффициента $\operatorname{Disp}_{\dot{\tau}}(N)$ для учета распределения суммарного сигнального воздействия между всеми клетками-мишенями. Подобный подход существенно отличается от классической схемы с обычным мажоритарным голосованием.

2. Специфика формализации базовой модели. Для оценки значений универсальных параметров $T, I_{S}, \operatorname{Pr}, D, O, K$ мы будем опираться на экспериментальные данные и предсказания моделей внутриклеточной биохимии (к примеру, из работы [17]). Рассмотрим простейшие примеры алгоритмов для оценки этих параметров.

Пример 1. Определение множества типов клеток $T$.

Шаг 1. Используя информацию о рассматриваемом организме и его тканях, мы получаем начальное приближение для множества типов $T$.

Шаг 2. Выбираем набор ключевых параметров, отвечающих за тип клетки на биохимическом уровне. К ним, в первую очередь, относятся белки, а также активные ${ }^{6}$ гены, специфичные для данных типов.

Шаг 3. Для выбранных на шаге 2 белков и генов вводится метрика, которая позволит проверить типы клеток на биохимическое «подобие». Отметим, что подобная метрика вводится на векторах из концентраций этих белков и состояний генов клеток вида $c=\left(x_{1}^{c}, \ldots, x_{k}^{c}\right)$. Простейший вариант задания такой метрики - метрика на основе весовых коэффициентов

$$
\rho\left(c_{1}, c_{2}\right)=\sum \alpha_{i}\left|x_{i}^{c_{1}}-x_{i}^{c_{2}}\right|,
$$

где $\alpha_{i}>0$ задают вклад отдельного белка или гена $x_{i}^{c}$ в определение типа с биохимической точки зрения.

Шаг 4. Проводим измерения концентраций белков и состояний генов для эталонных клеток всех типов из начального приближения для множества $T$.

\footnotetext{
${ }^{6}$ Варианты определения данного понятия разобраны в работе [17].
} 
Осуществляем кластеризацию этих данных алгоритмом FOREL. Kaждый отдельный кластер считается отдельным типом клеток, а формальный элемент центра кластера выбирается в качестве эталонного представителя клеток данного типа.

Пример 2. Определение интенсивности синтеза сигналов $I_{S}(\tau)$.

Шаг 1. Для всех типов клеток $\tau$ определяем, какие виды реальных сигналов эти клетки способны вырабатывать $\left\{S_{1}(\tau), \ldots, S_{k}(\tau)\right\}$.

Шаг 2. Выбираем контрольное время $\Delta t$, за которое клеткой будут синтезированы все сигналы из этого набора $\left\{S_{1}(\tau), \ldots, S_{k}(\tau)\right\}$.

Шаг 3. Оцениваем, сколько сигналов $\left\{N_{S_{1}}(\tau), \ldots, N_{S_{k}}(\tau)\right\}$ каждого вида клетка выделит за контрольное время $\Delta t$. Количество сигналов в единицу времени можно будет посчитать как $I_{j}(\tau)=N_{S_{j}}(\tau) / \Delta t$.

Шаг 4. В итоге получаем оценку для параметра $I_{S}(\tau)=\min _{j} I_{j}(\tau)$.

ЗАмечАние 5 . Отметим, что оценку числа сигналов $N_{j}(\tau)$ с помощью биохимической модели можно производить только в том случае, если в этой модели учитываются признаки экспорта молекул за пределы клеточной мембраны. Если такой механизм не учтен в модели, то единственный вариант оценки $N_{j}(\tau)$ - их экспериментальное измерение.

ПримеР 3. Определение приоритетов сигналов $\operatorname{Pr}\left(\tau, \tau_{*}\right)$.

Шаг 1. Для всех химических сигналов из набора $\left\{S_{1}\left(\tau_{*}\right), \ldots, S_{k}\left(\tau_{*}\right)\right\}$, определенных для типа $\tau_{*}$ на шаге 2 из примера 2 , фиксируется суммарное число реакций $B\left(\tau, S_{j}\left(\tau_{*}\right)\right)$ в единицу времени, которые катализируются под действием вторичных посредников от каждого сигнала $S_{j}\left(\tau_{*}\right)$ внутри клетки типа $\tau$.

Шаг 2. Пользуясь данными о количестве сигналов $\left\{N_{S_{1}}(\tau), \ldots, N_{S_{k}}(\tau)\right\}$, полученными на шаге 3 в примере 2, проводим оценку приоритетов по формуле

$$
\operatorname{Pr}\left(\tau, \tau_{*}\right)=\frac{1}{\sum_{j} N_{S_{j}}\left(\tau_{*}\right)} \sum_{j=1}^{k} N_{S_{j}}\left(\tau_{*}\right) B\left(\tau, S_{j}\left(\tau_{*}\right)\right)
$$

Пример 4. Определение параметров деления клеток $D\left(\tau, \tau^{*}\right)$ и $O\left(\tau, \tau^{*}\right)$.

Шаг 1 . Либо экспериментально воспроизводим прием клеткой типа $\tau$ всех сигналов из набора $\left\{S_{1}\left(\tau_{*}\right), \ldots, S_{k}\left(\tau_{*}\right)\right\}$, либо воспроизводим его с помощью биохимической модели.

Шаг 2. Либо дожидаемся деления клетки $\tau$, либо воспроизводим его с помощью биохимической модели. Оцениваем значения концентраций ключевых белков и состояния генных сетей $c=\left(x_{1}^{c}, \ldots, x_{k}^{c}\right)$ для обеих дочерних клеток.

Шаг 3. Проводим оценку типов дочерних клеток, используя данные, полученные при кластеризации векторов $\left(x_{1}^{c}, \ldots, x_{k}^{c}\right)$ в примере 1.

- В случае, если одна из дочерних клеток со своим вектором концентраций белков и состояний генов попадает в кластер для типа $\tau$, то считаем деление асимметричным $O\left(\tau, \tau^{*}\right)=1$. 
- В случае, если для второй дочерней клетки получаем, что вектор $c=\left(x_{1}^{c}, \ldots, x_{k}^{c}\right)$ для нее не попадает ни в один кластер, то добавляем в множество $T$ новый тип $\tau_{n e w}$ и сохраним значение вектора c как центр этого нового кластера.

- Если кластер для второй дочерней клетки соответствует типу $\tau_{c}$, то положим значение $\tau_{c}=D\left(\tau, \tau_{*}\right)$, где $\tau_{c}$ входит в потенциально расширенное множество типов $T$.

ПримеР 5. Определение элементарных приращений $K\left(\tau, \tau^{*}\right)$.

Шаг 1. Фиксируем время $t_{d}$, за которое клетки типа $\tau$ в отсутствие раздражения от $\tau^{*}$ приступают к делению или апоптозу. В случае, если такое событие не наблюдается на достаточно длительном промежутке наблюдения, полагается $t_{0}=\infty$.

Шаг 2. Экспериментально или с помощью биохимической модели воспроизводим прием клеткой типа $\tau$ всех сигналов из набора $\left\{S_{1}\left(\tau_{*}\right), \ldots, S_{k}\left(\tau_{*}\right)\right\}$. Количество сигналов возьмем равным $N_{S_{j}}$, как на шаге 3 в примере 2 , оцененных для контрольного времени $\Delta t$. Фиксируем время $t_{d}^{*}$, за которое клетки типа $\tau$ в этом случае будут приступать к делению или апоптозу.

Шаг 3. Пользуясь упрощающим положением, что ускорение деления осуществлялось за промежуток, сравнимый с временем $\Delta t$, получаем следующую формулу для оценки параметра $K\left(\tau, \tau^{*}\right)$ :

$$
K\left(\tau, \tau^{*}\right)=\frac{1 / t_{d}^{*}-1 / t_{d}}{\Delta t}=\frac{t_{d}-t_{d}^{*}}{t_{d}^{*} \Delta t t_{d}} .
$$

При использовании биохимической модели время наступления деления можно определить косвенно по прохождению клеткой контрольных точек клеточного цикла. Подобное событие в рамках биохимической модели может быть описано как выход концентраций определенных белков за пределы некоторых контрольных уровней. В случае, если белок выступает в роли ингибитора для прохождения контрольной точки клеточного цикла, то нас будет интересовать тот момент времени, когда его концентрация будет опускаться ниже контрольного уровня. Если же белок выступает в роли катализатора для прохождения контрольной точки клеточного цикла, то нас будет интересовать тот момент времени, когда его концентрация будет подниматься выше контрольного уровня.

Заключение. Использование альтернативного пути для формализации параметров модели (1)-(10) на основе экспериментальных данных или биохимической модели позволяет абстрагироваться от типа модели, и задействовать при желании те же значения параметров в дискретных имитационных моделях. В результате при использовании универсальных параметров мы получаем следующее разделение функций между моделями разных классов:

1) биохимические модели - формализация параметров;

2) популяционные модели - проверка формализации на адекватность, поиск скрытых параметров, исследование динамических режимов;

3) имитационные модели - визуализация клеточных скоплений в трехмерном пространстве, наглядное воспроизведение морфогенеза. 
Аналогичный результат также можно получить и для других типов моделей, подобрав для них подходящие наборы универсальных параметров. В частности, можно ввести активные и пассивные механические напряжения в ткани, а их воздействие на клетки представить в виде генерации вторичных сигнальных молекул внутри клеток (подробный обзор этих механизмов см. в работе [18]). В этом случае можно провести анализ отклика клетки с помощью биохимической модели и сравнить воздействие механических раздражений с действием молекул морфогенов.

Главным ограничивающим фактором для применения модели (1)-(10) является сложность формализации ее параметров, поскольку она требует получения специфических экспериментальных данных, которые на данный момент практически никем не измеряются. Не менее сложным является и получение данных о численности клеток как функциях $N(\tau)$ от их типов $\tau$ для отдельных тканей на основе трехмерных томографий этих тканей. Фактически данная задача может быть решена средствами машинного обучения, но для ее эффективного решения необходимо наличие доступных баз данных по томографиям исследуемых тканей. Если допустить, что обе эти проблемы устранены, то тогда базовую модель (1)-(10) можно было бы задействовать для моделирования тканей растений, а вот для тканей животных она представляется слишком грубым приближением. Это связано с тем, что для растений в отличие от животных характерна более высокая статичность тканей, и поэтому потребность в моделировании механических воздействий и миграции клеток не столь ярко выражена.

Конкурирующие интересы. У меня нет конкурирующих интересов.

Авторская ответственность. Я несу полную ответственность за предоставление окончательной версии рукописи в печать. Окончательная версия рукописи мною одобрена.

Финансирование. Исследование не имело финансирования.

\section{Библиографический список}

1. Urdy S. Principles of morphogenesis: the contribution of cellular automata models (Book Review) // Acta Zoologica, 2009. vol.90, no. 2. pp. 205-208. doi: 10.1111/j.1463-6395. 2008.00333.x.

2. Palsson E. A three-dimensional model of cell movement in multicellular systems // Future Generation Computer Systems, 2001. vol.17, no.7. pp. 835-852. doi:10.1016/ S0167-739X (00) 00062-5.

3. Drasdo D., Höhme S. A single-cell-based model of tumor growth in vitro: monolayers and spheroids // Physical Biology, 2005. vol. 2, no. 3. pp. 133-147. doi: 10.1088/1478-3975/2/ $3 / 001$.

4. Drasdo D. Center-based Single-cell Models: An Approach to Multi-cellular Organization Based on a Conceptual Analogy to Colloidal Particles / Single-Cell-Based Models in Biology and Medicine/ Mathematics and Biosciences in Interaction. Basel: Birkhäuser, 2007. pp. 171-196. doi : 10.1007/978-3-7643-8123-3_8.

5. Bauer A. L., Jackson T. L., Jiang Y. A cell-based model exhibiting branching and anastomosis during tumor-induced angiogenesis // Biophysical Journal, 2007. vol. 92, no. 9. pp. 31053121. doi : 10.1529/biophysj.106.101501.

6. Hirashima T., Iwasa Y., Morishita Y. Dynamic modeling of branching morphogenesis of ureteric bud in early kidney development // Journal of Theoretical Biology, 2009. vol. 259, no. 1. pp. 58-66. doi: 10.1016/j.jtbi.2009.03.017. 
7. Szabó A., Czirók A. The Role of Cell-Cell Adhesion in the Formation of Multicellular Sprouts // Math. Model. Nat. Phenom., 2010. vol.5, no. 1. pp. 106-122. doi: 10.1051/mmnp/ 20105105.

8. Taber L. A. Towards a unified theory for morphomechanics // Philos. Trans. Ser. A, 2009. vol. 367, no. 1902. pp. 3555-3583. doi: 10.1098/rsta.2009.0100.

9. Wyczalkowski M. A., Chen Z., Filas B. A., Varner V. D., Taber L. A. Computational models for mechanics of morphogenesis // Birth Defects Res. C, 2012. vol.96, no. 2. pp. 132-152. doi : 10.1002/bdrc.21013.

10. Forgacs G., Foty R. A., Shafrir Y., Steinberg M. S. Viscoelastic properties of living embryonic tissues: a quantitative study // Biophysical Journal, 1998. vol. 74, no. 5. pp. 2227-2234. doi : 10.1016/S0006-3495(98)77932-9.

11. Ranft J., Basan M., Elgeti J., Joanny J.-F., Prost J., Jülicher F. Fluidization of tissues by cell division and apoptosis // Proc. Natl. Acad. Sci. USA, 2010. vol. 107, no. 49. pp. 2086320868. doi : 10.1073/pnas.1011086107.

12. Dillon R., Othmer H. G. A Mathematical Model for Outgrowth and Spatial Patterning of the Vertebrate Limb Bud // Journal of Theoretical Biology, 1999. vol. 197, no. 3. pp. 295-330. doi: 10.1006/jtbi.1998.0876.

13. Keller E. F., Segel L. A. Initiation of slime mold aggregation viewed as an instability // Journal of Theoretical Biology, 1970. vol.26, no.3. pp. 399-415. doi: 10.1016/0022-5193(70) 90092-5.

14. Tanaka S. Simulation Frameworks for Morphogenetic Problems // Computation, 2015. vol. 3, no. 2. pp. 197-221. doi: 10.3390/computation3020197.

15. Brauer F., Castillo-Chavez C. Mathematical Models in Population Biology and Epidemiology / Texts in Applied Mathematics. vol. 40. New York: Springer Verlag, 2012. xxiv +508 pp. doi : $10.1007 / 978-1-4614-1686-9$.

16. Назаров М. Н. Моделирование роста ткани с учётом возможности внешнего воздействия на её форму // ПДМ, 2013. № 4(22). С. 103-113.

17. Назаров М. Н. Базовая математическая модель для описания процессов регуляции биосинтеза белков // Вестн. Удмуртск. ун-та. Матем. Мех. Компъют. науки, 2016. T. 26, № 4. C. 515-524. doi : 10.20537/vm160406.

18. Finch-Edmondson M., Sudol M. Framework to function: mechanosensitive regulators of gene transcription// Cellular and Molecular Biology Letters, 2016. vol.21, 28. 23 pp. doi: 10. 1186/s11658-016-0028-7. 
MSC: 35Q92; 92B05, 92D25

\title{
Mathematical modelling of tissue formation on the basis of ordinary differential equations
}

\author{
M. N. Nazarov \\ National Research University of Electronic Technology, \\ 1, Shokin square, Zelenograd, Moscow, 124498, Russian Federation
}

\begin{abstract}
A mathematical model is proposed for describing the population dynamics of cellular clusters on the basis of systems of the first-order ordinary differential equations. The main requirement for the construction of model equations was to obtain a formal biological justification for their derivation, as well as proof of their correctness. In addition, for all the parameters involved in the model equations, the presence of biological meaning was guaranteed, as well as the possibility of evaluating them either during the experiment or by using models of intracellular biochemistry. In the desired model the intercellular exchange of a special signal molecules was chosen as the main mechanism for coordination of the tissue growth and new types selection during cell division. For simplicity, all signalling molecules that can create cells of the same type were not considered separately in the model, but were instead combined in a single complex of molecules: a 'generalized signal'. Such an approach allows us to eventually assign signals as a functions of cell types and introduce their effects in the form of matrices in the models, where the rows are responsible for the types of cells receiving the signals, and the columns for the types of cells emitting signals.
\end{abstract}

Keywords: morphogenesis modeling, ordinary differential equations, system biology, hierarchical models.

Received: $22^{\text {nd }}$ March, 2017 / Revised: 13 $3^{\text {th }}$ June, $2017 /$

Accepted: $18^{\text {th }}$ September, $2017 /$ First online: $20^{\text {th }}$ September, 2017

Competing interests. I have no competing interests.

Author's Responsibilities. I take full responsibility for submitting the final manuscript in print. I approved the final version of the manuscript.

Funding. The research has not had any funding.

\section{Article}

ㅇ ()(i) The content is published under the terms of the Creative Commons Attribution 4.0 International License (http://creativecommons.org/licenses/by/4.0/)

Please cite this article in press as:

N a z a r ov M. N. Mathematical modelling of tissue formation on the basis of ordinary differential equations, Vestn. Samar. Gos. Tekhn. Univ., Ser. Fiz.-Mat. Nauki [J. Samara State Tech. Univ., Ser. Phys. Math. Sci.], 2017, vol. 21, no. 3, pp. 581-594. doi: 10.14498/vsgtu1535 (In Russian).

\section{Author's Details:}

Maxim N. Nazarov (D) http://orcid.org/0000-0001-8064-1374

Senior Lecturer; Dept. of Higher Mathematics-1; e-mail: nazarov-maximilian@yandex.ru 


\section{References}

1. Urdy S. Principles of morphogenesis: the contribution of cellular automata models (Book Review), Acta Zoologica, 2009, vol. 90, no. 2, pp. 205-208. doi : 10.1111/j.1463-6395. 2008. 00333.x.

2. Palsson E. A three-dimensional model of cell movement in multicellular systems, $F u$ ture Generation Computer Systems, 2001, vol.17, no.7, pp. 835-852. doi:10.1016/ S0167-739X (00) 00062-5.

3. Drasdo D., Höhme S. A single-cell-based model of tumor growth in vitro: monolayers and spheroids, Physical Biology, 2005, vol. 2, no. 3, pp. 133-147. doi : 10.1088/1478-3975/2/3/ 001.

4. Drasdo D. Center-based Single-cell Models: An Approach to Multi-cellular Organization Based on a Conceptual Analogy to Colloidal Particles, In: Single-Cell-Based Models in Biology and Medicine, Mathematics and Biosciences in Interaction. Basel, Birkhäuser, 2007, pp. 171-196. doi: 10.1007/978-3-7643-8123-3_8.

5. Bauer A. L., Jackson T. L., Jiang Y. A cell-based model exhibiting branching and anastomosis during tumor-induced angiogenesis, Biophysical Journal, 2007, vol. 92, no. 9, pp. 31053121. doi: 10.1529/biophysj.106.101501.

6. Hirashima T., Iwasa Y., Morishita Y. Dynamic modeling of branching morphogenesis of ureteric bud in early kidney development, Journal of Theoretical Biology, 2009, vol. 259, no. 1, pp. 58-66. doi: 10.1016/j.jtbi.2009.03.017.

7. Szabó A., Czirók A. The Role of Cell-Cell Adhesion in the Formation of Multicellular Sprouts, Math. Model. Nat. Phenom., 2010, vol.5, no. 1, pp. 106-122. doi: 10.1051/mmnp/ 20105105.

8. Taber L. A. Towards a unified theory for morphomechanics, Philos. Trans. Ser. A, 2009, vol. 367, no. 1902, pp. 3555-3583. doi: 10.1098/rsta.2009.0100.

9. Wyczalkowski M. A., Chen Z., Filas B. A., Varner V. D., Taber L. A. Computational models for mechanics of morphogenesis, Birth Defects Res. C, 2012, vol.96, no. 2, pp. 132-152. doi: 10.1002/bdrc.21013.

10. Forgacs G., Foty R. A., Shafrir Y., Steinberg M. S. Viscoelastic properties of living embryonic tissues: a quantitative study, Biophysical Journal, 1998, vol.74, no. 5, pp. 2227-2234. doi : 10.1016/S0006-3495(98)77932-9.

11. Ranft J., Basan M., Elgeti J., Joanny J.-F., Prost J., Jülicher F. Fluidization of tissues by cell division and apoptosis, Proc. Natl. Acad. Sci. USA, 2010, vol. 107, no. 49, pp. 2086320868. doi: 10.1073/pnas. 1011086107.

12. Dillon R., Othmer H. G. A Mathematical Model for Outgrowth and Spatial Patterning of the Vertebrate Limb Bud, Journal of Theoretical Biology, 1999, vol.197, no. 3, pp. 295-330. doi: 10.1006/jtbi.1998.0876.

13. Keller E. F., Segel L. A. Initiation of slime mold aggregation viewed as an instability, Journal of Theoretical Biology, 1970, vol.26, no. 3, pp. 399-415. doi: 10.1016/0022-5193(70) 90092-5.

14. Tanaka S. Simulation Frameworks for Morphogenetic Problems, Computation, 2015, vol. 3, no. 2, pp. 197-221. doi: 10.3390/computation3020197.

15. Brauer F., Castillo-Chavez C. Mathematical Models in Population Biology and Epidemiology, Texts in Applied Mathematics, vol. 40. New York, Springer Verlag, 2012, xxiv +508 pp. doi: 10.1007/978-1-4614-1686-9.

16. Nazarov M. N. Modelling the tissue growth with the possibility of external influence on tissue shape, Prikl. Diskr. Mat., 2013, no.4(22), pp. 103-113 (In Russian).

17. Nazarov M. N. The basic mathematical model for the description of regulatory processes of protein biosynthesis, Vestn. Udmurtsk. Univ. Mat. Mekh. Komp. Nauki, 2016, vol. 26, no. 4, pp. 515-524 (In Russian). doi: 10.20537/vm160406.

18. Finch-Edmondson M., Sudol M. Framework to function: mechanosensitive regulators of gene transcription, Cellular and Molecular Biology Letters, 2016, vol. 21, 28, 23 pp. doi: 10.1186/ s11658-016-0028-7. 